\title{
JARID1B promotes metastasis and epithelial-mesenchymal transition via PTEN/AKT signaling in hepatocellular carcinoma cells
}

This article has been corrected. Correction in: Oncotarget. 2020; 11:1797-1798.

Bo Tang ${ }^{1,2}$, Guangying $\mathbf{Q i}^{2,3}$, Fang Tang ${ }^{1,2}$, Shengguang Yuan ${ }^{1,2}$, Zhenran Wang ${ }^{1,2}$, Xingsi Liang ${ }^{1,2}$, Bo Li ${ }^{1,2}$, Shuiping Yu ${ }^{1,2}$, Jie Liü ${ }^{1,2}$, Qi Huang ${ }^{1,2}$, Yangchao Wei ${ }^{1,2}$, Run Zhai ${ }^{1,2}$, Biao Lei ${ }^{1,2}$, Hongping Yư ${ }^{4}$, Xingyuan $\mathrm{Jiao}^{5}$ and Songqing $\mathrm{He}^{1,2}$

${ }^{1}$ Department of Hepatobiliary Surgery, Guilin Medical University, Affiliated Hospital, Guilin, Guangxi, People's Republic of China

${ }^{2}$ Laboratory of Liver Injury and Repair Molecular Medicine, Guilin Medical University, Guilin, Guangxi, People's Republic of China

${ }^{3}$ Department of Pathology and Physiopathology, Guilin Medical University, Guilin, Guangxi, People's Republic of China

${ }^{4}$ Department of Epidemiology and Statistics, School of Public Health, Guilin Medical College, Guilin, Guangxi, People's Republic of China

${ }^{5}$ Department of General Surgery, The First Affiliated Hospital, Sun Yat-Sen University, Guangzhou, People's Republic of China

Correspondence to: Songqing He, email: hesongqingdoc@yeah.net

Xingyuan Jiao, email: xyjdoctor@163.com

Keywords: JARIDIB, metastasis, epithelial-mesenchymal transition, invasion, PTEN

Received: February 16, $2015 \quad$ Accepted: March 10, $2015 \quad$ Published: March 30, 2015

This is an open-access article distributed under the terms of the Creative Commons Attribution License, which permits unrestricted use, distribution, and reproduction in any medium, provided the original author and source are credited.

\section{ABSTRACT}

JARID1B is a member of the family of $\mathrm{JmjC}$ domain-containing proteins that removes methyl residues from methylated lysine 4 on histone H3 lysine 4 (H3K4). JARID1B has been proposed as an oncogene in many types of tumors; however, its role and underlying mechanisms in hepatocellular carcinoma (HCC) remain unknown. Here we show that JARID1B is elevated in HCC and its expression level is positively correlated with metastasis. In addition Kaplan-Meier survival analysis showed that high expression of JARID1B was associated with decreased overall survival of HCC patients. Overexpression of JARID1B in HCC cells increased proliferation, epithelialmesenchymal transition, migration and invasion in vitro, and enhanced tumorigenic and metastatic capacities in vivo. In contrast, silencing JARID1B in aggressive and invasive HCC cells inhibited these processes. Mechanistically, we found JARID1B exerts its function through modulation of $\mathrm{H} 3 \mathrm{~K} 4 \mathrm{me} 3$ at the PTEN gene promoter, which was associated with inactive PTEN transcription. PTEN overexpression blocked JARID1B-driven proliferation, EMT, and metastasis. Our results, for the first time, portray a pivotal role of JARID1B in stimulating metastatic behaviors of HCC cells. Targeting JARID1B may thus be a useful strategy to impede HCC cell invasion and metastasis.

\section{INTRODUCTION}

The incidence of hepatocellular carcinoma (HCC) has dramatically increased in Western countries and has become more prevalent in Asian countries [1,2]. Although significant improvement in diagnosis and treatment of HCC has been achieved, the outcome of patients remains dismal with a median survival of merely a few months [3]. This poor prognosis is due to high recurrent and metastatic rates even after the use of current treatment modalities [3]. Furthermore, biomarkers that are currently used clinically to predict the prognosis of HCC patients after curative surgical resection remain unsatisfactory in terms of both accuracy and reproducibility. Therefore, it 
remains clinically important to identify novel prognostic biomarkers to improve the diagnosis and treatment of HCC patients.

Jumonji AT-rich interactive domain 1B (JARID1B) is a member of the family of JmjC domain-containing proteins that specifically removes methyl residues from tri-, di-, and monomethylated lysine 4 on histone $\mathrm{H} 3$ lysine 4 (H3K4) that are associated with active genes [4, 5]. JARID1B is a transcriptional repressor that harbors intrinsic histone demethylase activity. Trimethylation at $\mathrm{H} 3 \mathrm{~K} 4$ is an important histone mark associated with actively transcribed genes, and JARID1B specifically demethylates $\mathrm{H} 3 \mathrm{~K} 4 \mathrm{me} 3$ to a transcriptionally inactive state [6]. JARID1B is up-regulated in multiple tumor cell lines [4, 7-11]. Furthermore, JARID1B promotes proliferation of cancer cells, and knockdown of JARID1B causes a significant delay in the G1/S transition [12, 13]. JARID1B appears to promote breast tumorigenesis through the specific repression of antiproliferative genes including the BRCA1 breast tumor suppressor gene [8]. Thus, JARID1B may act as an oncogene, but whether JARID1B plays a role in HCC formation and metastasis remains unknown.

The vast majority of HCC patients succumb to their disease as a result of metastasis [14]. Currently, treatment options for metastatic HCC are limited and ineffective. Therefore, tremendous effort has been focused on the understanding of the mechanisms by which metastasis occurs in order to provide a more rational approach in the development of future metastatic hepatocellular carcinoma treatments. However, how metastases are formed remains less understood. Mounting evidence shows that in epithelial cancers, including HCC, induction of epithelialmesenchymal transition (EMT) is a major event that provides mobility to cancer cells in order to generate metastases $[15,16]$. EMT is characterized by the loss of epithelial characteristics and acquisition of a mesenchymal phenotype, which confers the ability for cancer cells to invade adjacent tissue and migrate to distant sites, where these cancer cells proliferate to generate new tumors [17]. Hence, clarifying the regulation of proliferation and EMT will greatly benefit our understanding of HCC metastasis.

In the current study, we show that JARID1B overexpression predicts poor prognosis in HCC patients undergoing curative resection. Additionally, we present the evidence that JARID1B expression promotes HCC cells growth, invasion, metastasis and EMT. These functional effects of JARID1B were exerted through control of PTEN transcriptional expression via $\mathrm{H} 3 \mathrm{~K} 4 \mathrm{me} 3$. The down-regulation of PTEN and upregulation of the PI3K pathway triggered by JARID1B therefore enforce HCC cells oncogenesis and metastasis. Our findings provide a novel mechanistic role of JARID1B in HCC metastasis, suggesting that JARID1B may serve as a potential therapeutic target for advanced HCCs.

\section{RESULTS}

\section{JARID1B is highly expressed and correlated with distant metastasis in $\mathrm{HCC}$}

To investigate whether JARID1B might be involved in HCC, the mRNA expression level of JARID1B in HCC tissues and its matched normal adjacent tissues was determined by qRT-PCR in 38 samples. As compared with normal tissues, HCC specimens showed overexpression of JARID1B (Figure 1A). We then analyzed JARID1B expression in HCCs without or with distant metastasis; we found that JARID1B mRNA overexpression was significantly correlated with distant metastasis in HCC tissues (Figure. 1C). The protein level of JARID1B in these tissue samples were also analyzed by western blot (Figure 1B). The protein level of JARID1B was upregulated in HCCs samples as compared with the normal adjacent tissues samples. Furthermore, we found that JARID1B protein expression was also significantly correlated with distant metastasis in HCC tissues (Figure. 1D). As showed in Supplemental Figure 1A and 1B, expression level of JARID1B protein and mRNA in mesenchymal HCC cell lines was higher than that in the epithelia HCC cell lines. These data demonstrated that the upregulation of JARID1B might be relevant to development of HCC.

We examined JARID1B protein expression in more HCC samples by IHC (Figure 2A). We observed that the level of JARID1B positive cells was markedly higher in HCC tissues than the level in the normal liver tissues (Figure 2B). Most importantly, JARID1B overexpression was consistently significantly correlated to distant metastasis in these HCC samples (Figure 2B). To investigate the relationship between JARID1B expression and clinicopathological parameters in the 178 cases with HCCs, these cases were first divided into two subgroups: "low JARID1B expression" and "high JARID1B expression" as defined in the immunohistochemistry section of "Materials and methods". Significant correlations were found between JARID1B expression and tumor diameter, microvascular invasion, and tumor differentiation. There were no statistical connections between JARID1B expression and the rest clinicopathological parameters, such as patient age, gender, and HBsAg (Table 1). The association between JARID1B expression in HCC and the survival time of selected patients was analyzed with Kaplan-Meier survival analysis (Figure 2C). The median overall survival time of high JARID1B expression group was significantly shorter than that of low JARID1B expression group $(P<$ $0.001)$. These results collectively indicate a functional role of JARID1B in aggressive behaviors of HCCs. 


\section{JARID1B promotes proliferative capacity of HCC cells}

In order to test the oncogenic activity of JARID1B in HCC, we retrovirally established stable overexpression of JARID1B in Huh7 and HepG2 cells, and silencing of JARID1B in SNU423 and SK-Hep1 cells. The levels of JARID1B in these resultant cell lines were verified by western blotting (Figure $3 \mathrm{~A}$ and $3 \mathrm{~B}$ ) and qRT-PCR (Supplemental Figure 2A and 2B). Compared to vectoronly controls, both Huh7-pBabe-JARID1B and HepG2pBabe-JARID1B cells had significant increases in cell proliferation by MTT assay (Figure 3C and 3D) and generated more numbers and larger colonies (Figure 3G and $3 \mathrm{H}$ ). In contrast, silencing of JARID1B in SNU423 and SK-Hep1 cells significantly reduced cell proliferation (Figure 3E and 3F) and clonogenicity (Figure 3I and 3J).
Consistent with these observations and previous reports $[8,23]$, we confirmed that cyclin $\mathrm{D} 1$, which promotes $\mathrm{G}(1)$ progression, was modulated upon JARID1B expression, JARID1B overexpression significantly increased the expression of Cyclin D1 while silencing JARID1B dramatically decreased its expression level (Figure 3K and $3 \mathrm{~L}$, Supplemental Figure $2 \mathrm{C}$ and 2D). To further understand and characterize the role of JARID1B in control of HCC cell growth, we analyzed the apoptotic activity of JARID1B in HCC cells. Annexin V binding assay showed that ectopic JARID1B expression reduced the cell proportion in apoptosis (Supplemental Figure 3A and $3 \mathrm{~B}$ ) and silencing JARID1B expression drastically increased the population of apoptotic cells (Supplemental Figure $3 \mathrm{C}$ and $3 \mathrm{D}$ ). Taking together, these results suggest that JARID1B is an important regulator of proliferation in HCC cells.

A

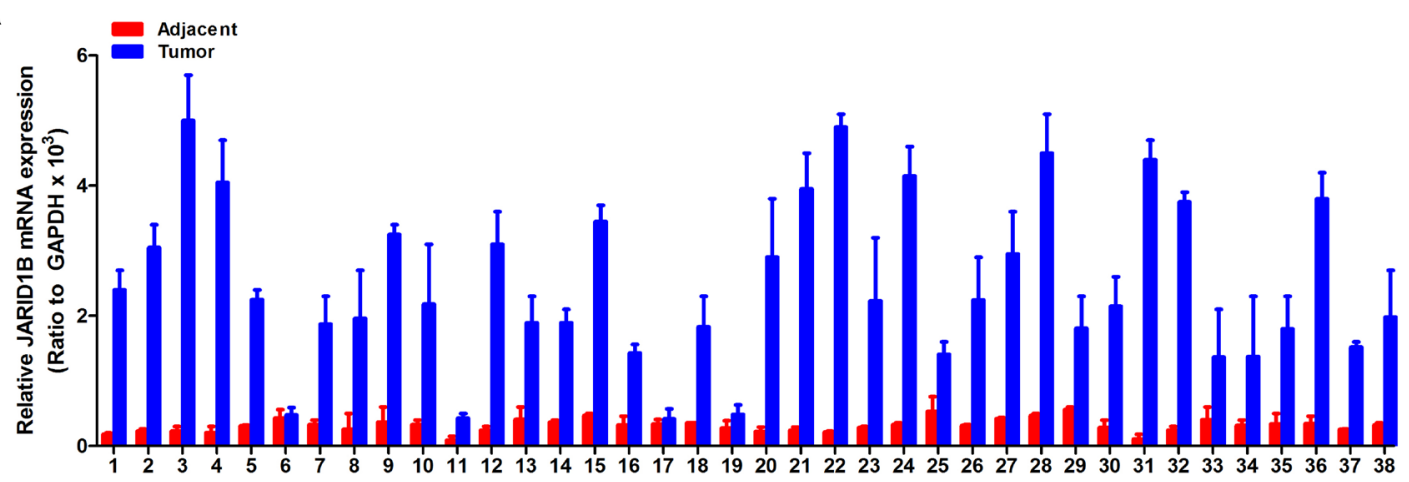

B

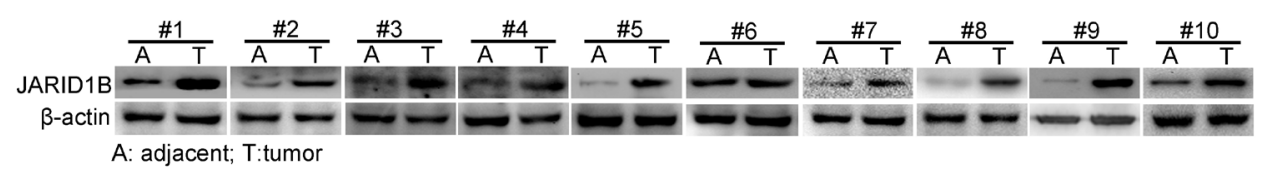

C

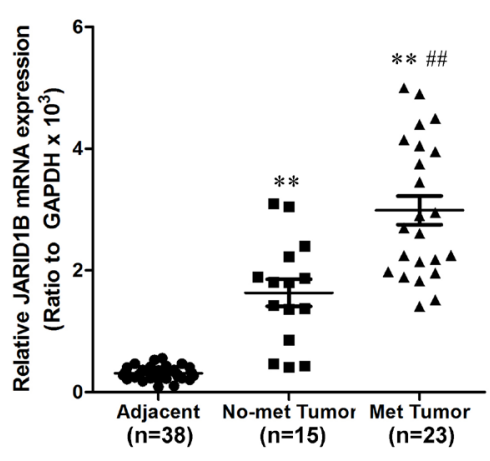

D

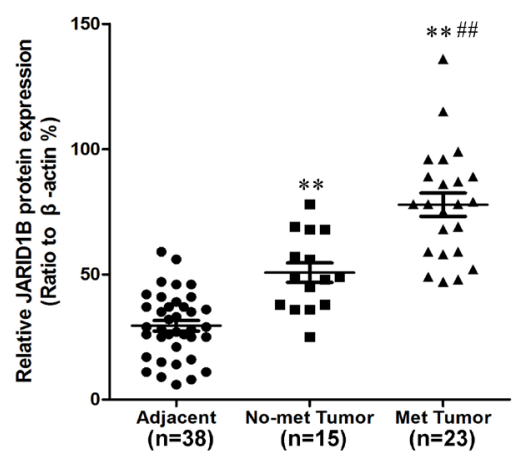

Figure 1: JARID1B is highly expressed in HCC. A, JARID1B mRNA expression was analyzed by quantitative RT-PCR in tumors and adjacent tissues. B, JARID1B protein expression was analyzed by Western blot in tumors and adjacent tissues and the representative results were shown. C, comparison of the expression levels of JARID1B mRNA in adjacent tissues, not metastasis and metastatic HCCs. D, comparison of the relative expression levels of JARID1B protein in adjacent tissues, not metastasis and metastatic HCCs. ${ }^{* *}$, compared with adjacent tissues $P<0.01$; \#, compared with not metastasis tissues $P<0.01$; Error bars, SD. 
Table 1: JARID1B staining and clinicopathologic characteristics of 178 hepatocellular carcinoma patients

\begin{tabular}{|c|c|c|c|c|}
\hline \multirow{2}{*}{ Variables } & \multicolumn{2}{|c|}{ JARID1B staining } & \multirow{2}{*}{ Total } & \multirow{2}{*}{$P^{\mathrm{a}}$} \\
\hline & Low & High & & \\
\hline \multicolumn{5}{|l|}{ Age (y) } \\
\hline$\leq 50$ & $53(74 \%)$ & $19(26 \%)$ & 72 & 0.084 \\
\hline$>50$ & $73(69 \%)$ & $33(31 \%)$ & 106 & \\
\hline \multicolumn{5}{|l|}{ Sex } \\
\hline Male & $119(79 \%)$ & $32(21 \%)$ & 151 & 0.399 \\
\hline Female & $22(81 \%)$ & $5(19 \%)$ & 27 & \\
\hline \multicolumn{5}{|l|}{ HBsAg } \\
\hline Negative & $39(83 \%)$ & $8(17 \%)$ & 47 & 0.094 \\
\hline Positive & $94(72 \%)$ & $37(28 \%)$ & 131 & \\
\hline \multicolumn{5}{|l|}{ HCV } \\
\hline Negative & $127(74 \%)$ & $45(26 \%)$ & 172 & 0.257 \\
\hline Positive & $5(83 \%)$ & $1(17 \%)$ & 6 & \\
\hline \multicolumn{5}{|l|}{ AFP } \\
\hline$\leq 20$ & $62(78 \%)$ & $17(22 \%)$ & 79 & 0.318 \\
\hline$>20$ & $71(72 \%)$ & $28(28 \%)$ & 99 & \\
\hline \multicolumn{5}{|c|}{$\gamma-\mathbf{G T}(\mathbf{U} / \mathbf{L})$} \\
\hline$\leq 54$ & $63(73 \%)$ & $21(27 \%)$ & 86 & 0.336 \\
\hline$>54$ & $73(79 \%)$ & $19(21 \%)$ & 92 & \\
\hline \multicolumn{5}{|c|}{ Liver cirrhosis } \\
\hline No & $24(77 \%)$ & $7(23 \%)$ & 31 & 0.362 \\
\hline Yes & $109(74 \%)$ & $38(26 \%)$ & 147 & \\
\hline \multicolumn{5}{|c|}{ Tumor diameter $(\mathrm{cm})$} \\
\hline$\leq 5$ & $101(82 \%)$ & $22(18 \%)$ & 123 & 0.026 \\
\hline$>5$ & $39(71 \%)$ & $16(29 \%)$ & 55 & \\
\hline \multicolumn{5}{|c|}{ Microvascular invasion } \\
\hline Absence & $62(83 \%)$ & $13(17 \%)$ & 75 & $<0.001$ \\
\hline Present & $58(56 \%)$ & $51(44 \%)$ & 103 & \\
\hline \multicolumn{5}{|c|}{ Tumor encapsulation } \\
\hline Complete & $65(75 \%)$ & $22(25 \%)$ & 87 & 0.268 \\
\hline None & $64(71 \%)$ & $26(29 \%)$ & 90 & \\
\hline \multicolumn{5}{|c|}{ Tumor differentiation } \\
\hline $\mathrm{I}+\mathrm{II}$ & $99(78 \%)$ & $28(22 \%)$ & 127 & 0.001 \\
\hline $\mathrm{III}+\mathrm{IV}$ & $31(61 \%)$ & $20(39 \%)$ & 51 & \\
\hline \multicolumn{5}{|l|}{ TNM stage } \\
\hline $\mathrm{I}$ & $115(75 \%)$ & $38(25 \%)$ & 153 & 0.446 \\
\hline $\mathrm{II}+\mathrm{III}$ & $19(76 \%)$ & $6(24 \%)$ & 25 & \\
\hline
\end{tabular}

Abbreviations: HBsAg, hepatitis B surface antigen; AFP, $\alpha$-fetoprotein; $\gamma$-GT, $\gamma$-glutamyl transferase; TNM, tumor-nodesmetastasis.

${ }^{a} p$-value $<0.05$ was considered statistically significant. $p$-values were calculated using the Pearson chi-square test. 


\section{JARID1B regulates the transition between epithelial and mesenchymal phenotypes in $\mathrm{HCC}$ cells}

To investigate whether JARID1B positively regulates cell migration and invasion, we first observed the morphological changes and found that both Huh7-pBabeJARID1B and HepG2-pBabe-JARID1B cells exhibited fibroblastic morphology (Figure 4A). This observation was further confirmed by expression analyses of epithelial and mesenchymal markers. We showed that JARID1B overexpression decreased the levels of epithelial markers (E-cadherin and $\alpha$-catenin) and increased the levels of mesenchymal markers (N-cadherin and vimentin) in both cell lines (Figure 4B and 4C). Conversely, both SNU423pSuper-shJARID1B and SK-Hep1-pSuper-shJARID1B cells reverted to an epithelial phenotype as compared to their respective control cells (Figure 4D). Consistent with this, silencing JARID1B increased levels of epithelial markers, and decreased levels of mesenchymal markers (Figure 4E and 4F). Taken together, these findings suggest that JARID1B plays an important role in regulating EMTMET plasticity of HCC cells.

A

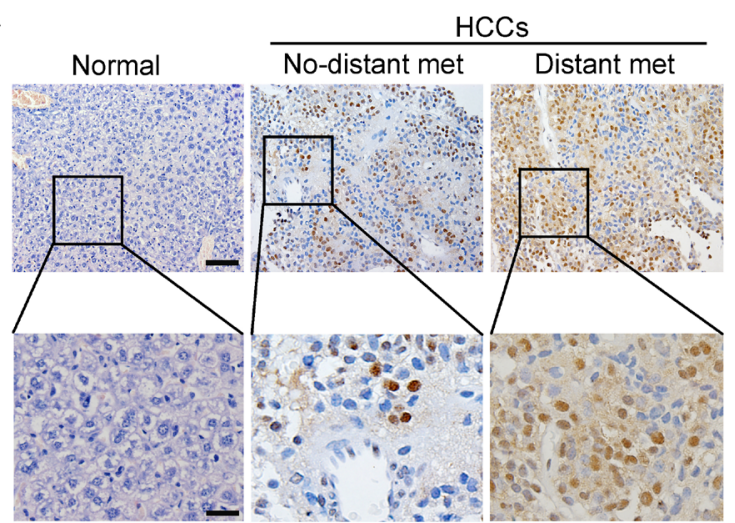

B

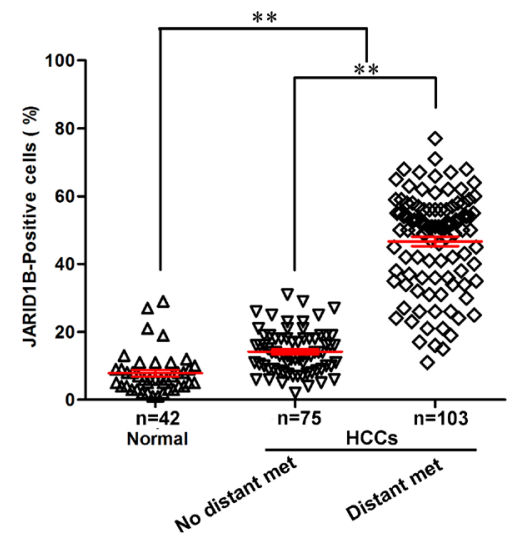

C

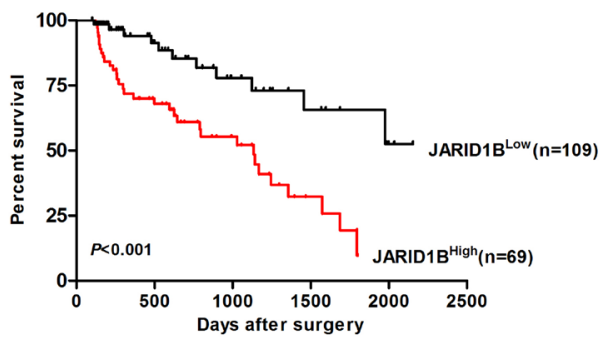

Figure 2: JARID1B is correlated with distant metastasis in HCC. A, JARID1B protein expression was analyzed by immunohistochemical analysis in 178 cases HCC tissues and the representative results were shown. B, semiquantification of JARID1B expression in normal tissues, primary HCC tissues without or with distant metastasis. Normal, normal liver tissues; no distant met, primary cancers without distant metastasis (in situ); distant met, primary cancers with distant metastasis. ${ }^{* *}, P<0.01$ is based on the Student $t$ test. Error bars, SD. C, the association between JARID1B expression in HCC and the survival time of selected patients was analyzed with Kaplan-Meier survival analysis. Scale bars, $50 \mu \mathrm{m}$ (upper) and $20 \mu \mathrm{m}$ (lower) in A 
A
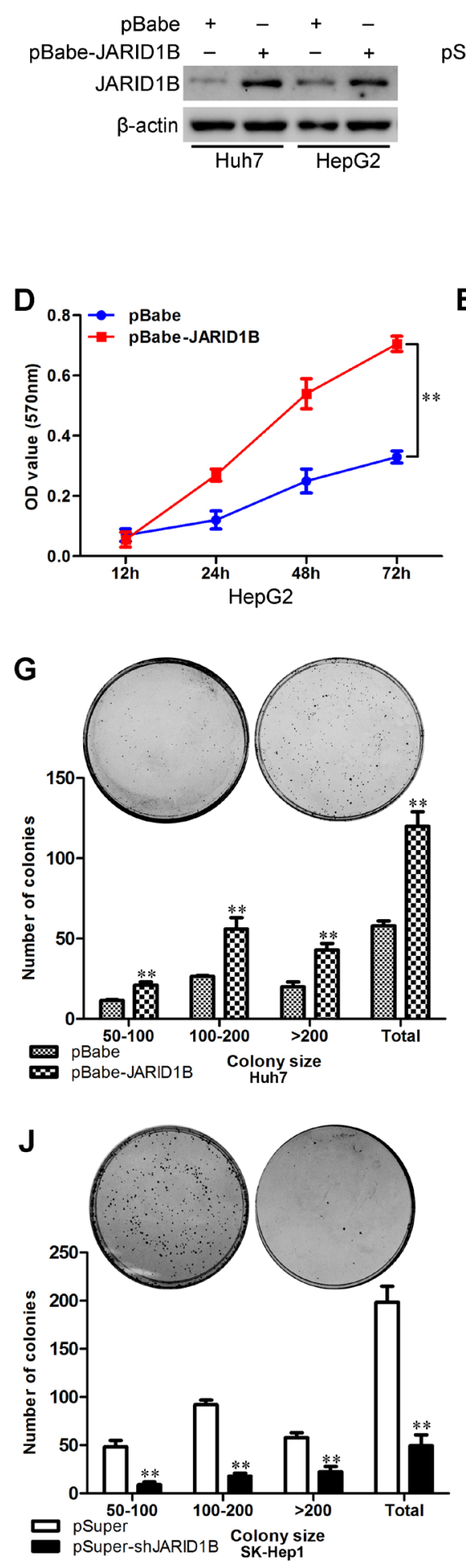

B

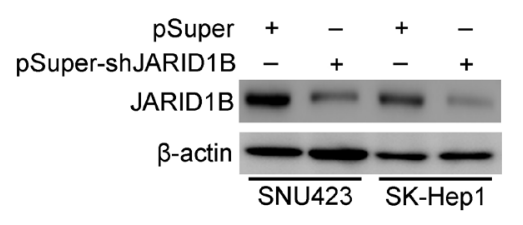

E
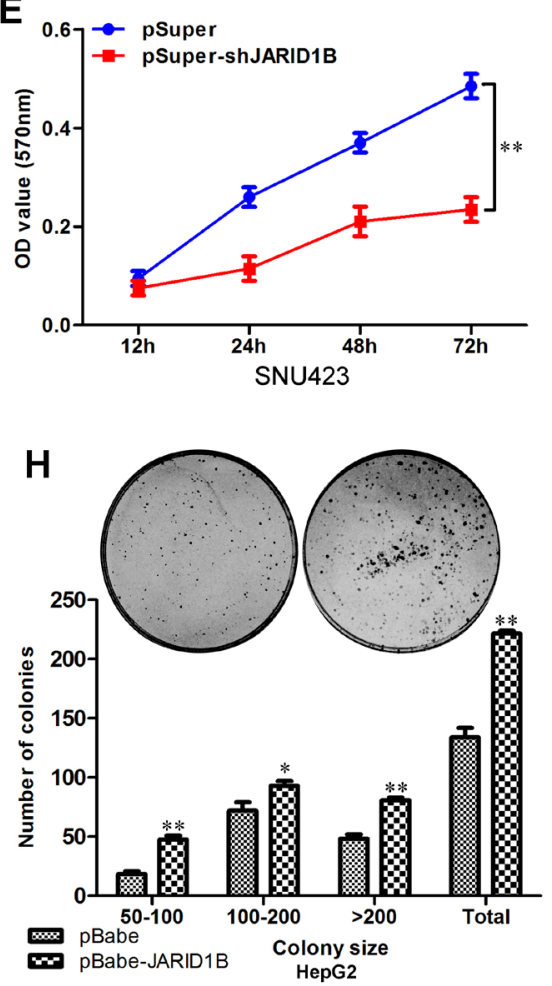

K

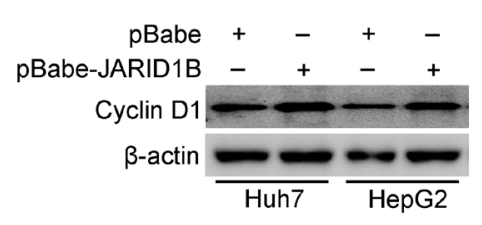

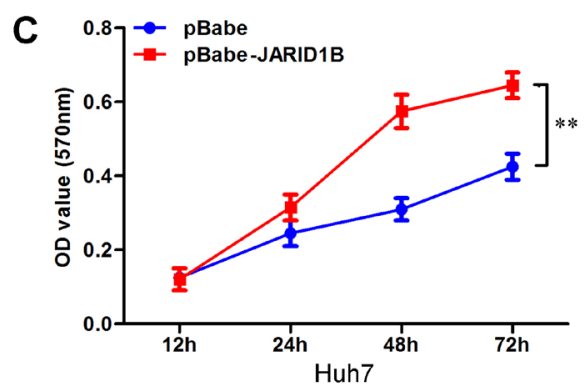

$\mathbf{F}$
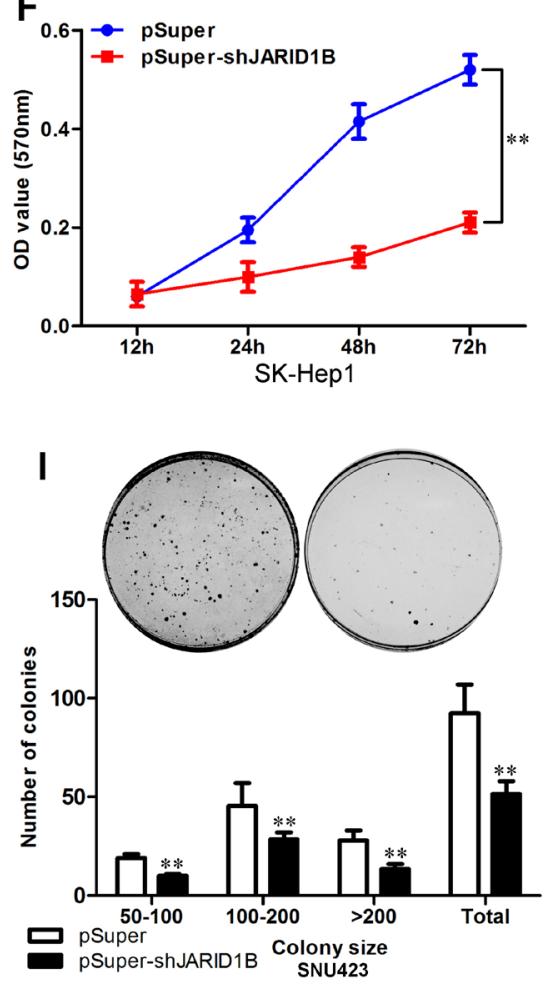

$\mathbf{L}$

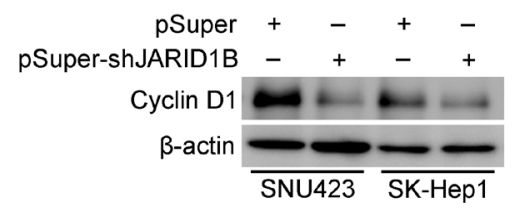

Figure 3: JARID1B promotes proliferative capacity of HCC cells. A and B, expression level of JARID1B was measured by Western blotting. C-F, Cell proliferation was examined by MTT assays. G-J, cell proliferation was examined by colony formation assays. $\mathbf{K}$ and $\mathbf{L}$, expression level of Cyclin D1 was measured by Western blotting. For colony formation assay, colonies containing more than 50 cells were counted and plotted; representative plates are shown in insets. ${ }^{* *}, P<0.01$ is based on the Student $t$ test. All results are from three independent experiments. Error bars, SD. 
A

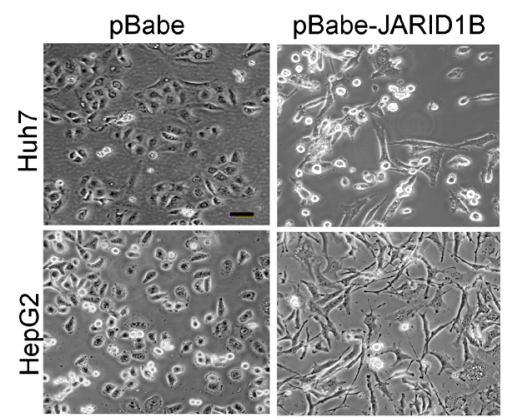

C

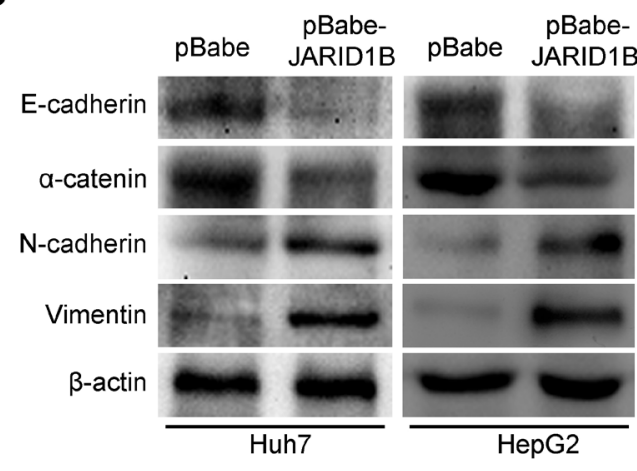

$\mathbf{E}$

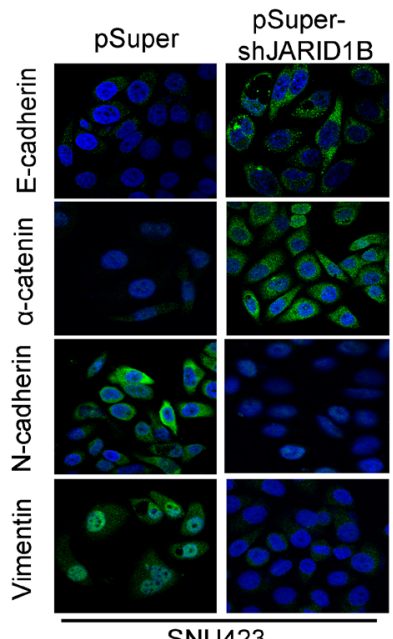

SNU423

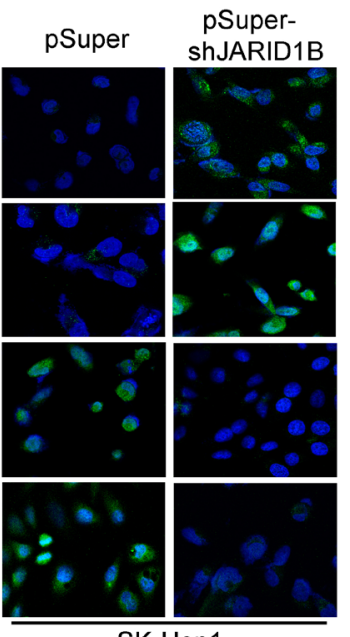

SK-Hep1
B

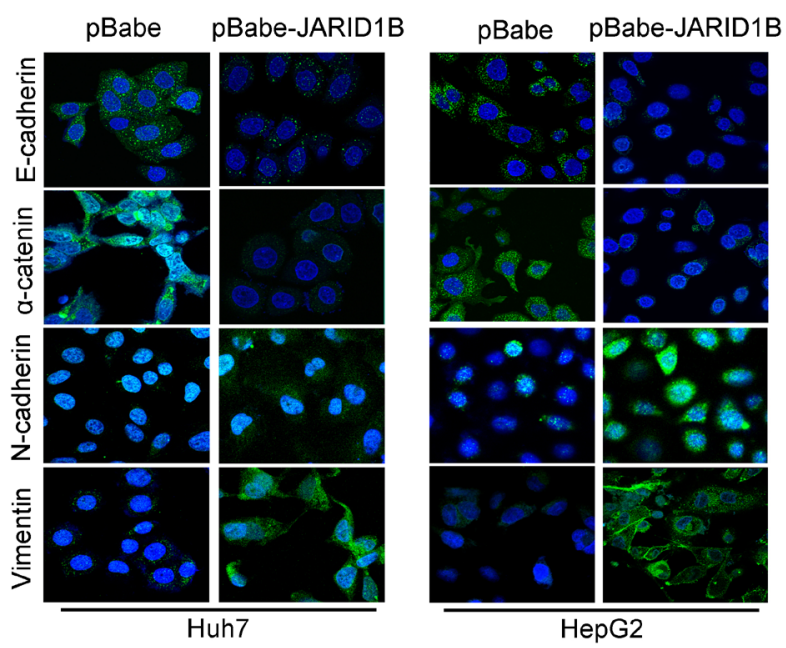

D

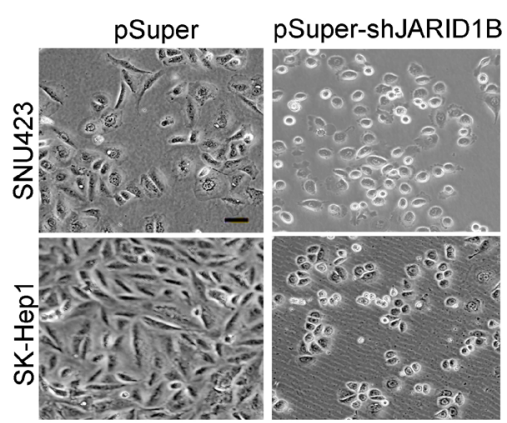

$\mathbf{F}$

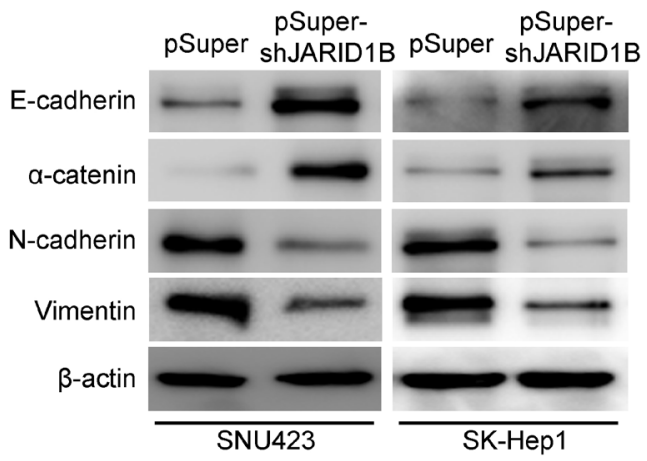

Figure 4: JARID1B regulates the transition between epithelial and mesenchymal phenotypes in HCC cells. A, representative phase-contrast images of Huh-7 and HepG2 cells showed JARID1B overexpression-modulated morphologic changes. B, expression of epithelial and mesenchymal marker was analyzed by immunofluorescence stains in Huh-7 and HepG2 cells. C, expression of epithelial and mesenchymal marker was analyzed by Western blotting in Huh-7 and HepG2 cells. D, representative phase-contrast images of SNU423 and SK-Hep1 cells showed JARID1B knockdown-modulated morphologic changes. E, expression of epithelial and mesenchymal marker was analyzed by immunofluorescence stains in SNU423 and SK-Hep1 cells. F, expression of epithelial and mesenchymal marker was analyzed by Western blotting in SNU423 and SK-Hep1 cells. 


\section{JARID1B promotes migratory and invasive capacities of $\mathrm{HCC}$ cells in vitro}

The effect of JARID1B on cell migration was first assessed by wound healing assay. Both Huh7pBabe-JARID1B and HepG2-pBabe-JARID1B cells had significantly faster closure of the wound area compared to their control cells (Figure 5A and 5B). This result was confirmed by Boyden's chamber assay (Figure $5 \mathrm{C}$ and
5D). Moreover, Huh7-pBabe-JARID1B and HepG2pBabe-JARID1B cells showed a greater degree of invasion through Matrigel (Figure 5C and 5D). In contrast, silencing JARID1B dramatically reduced the migratory and invasive capacity of SNU423 and SK-Hep1 cells (Figure 5E - H), suggesting that restoration of an epithelial phenotype through MET may dampen or inhibit their mobility potential. These results indicate that JARID1B promotes migratory and invasive behaviors in $\mathrm{HCC}$ cells.
A

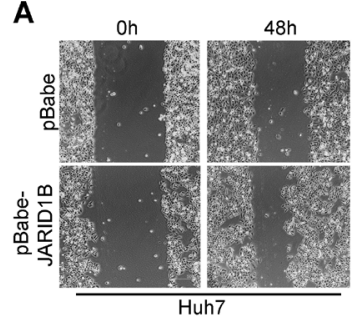

C

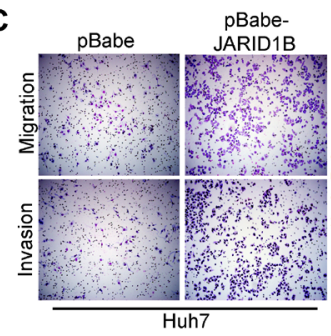

E

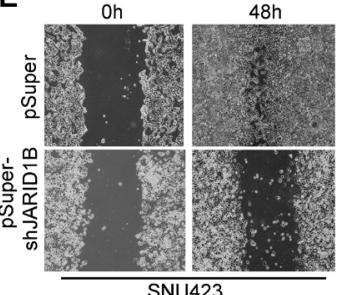

$48 \mathrm{~h}$

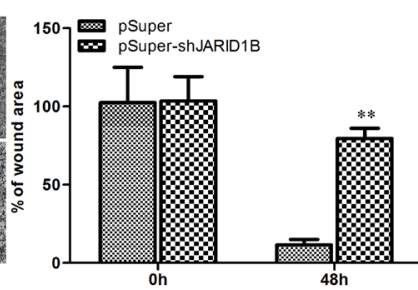

G
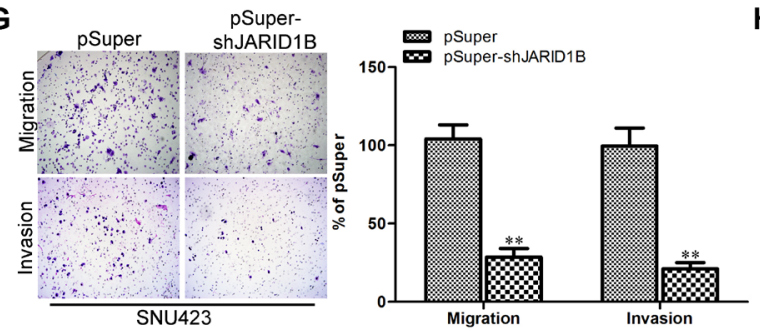

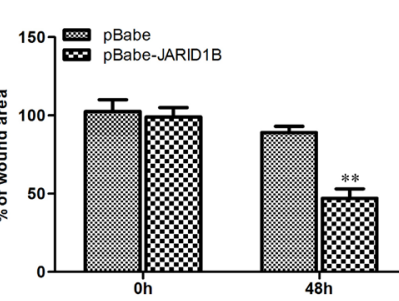

B
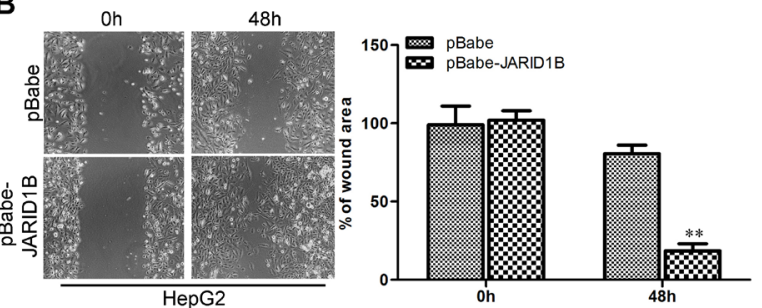

D
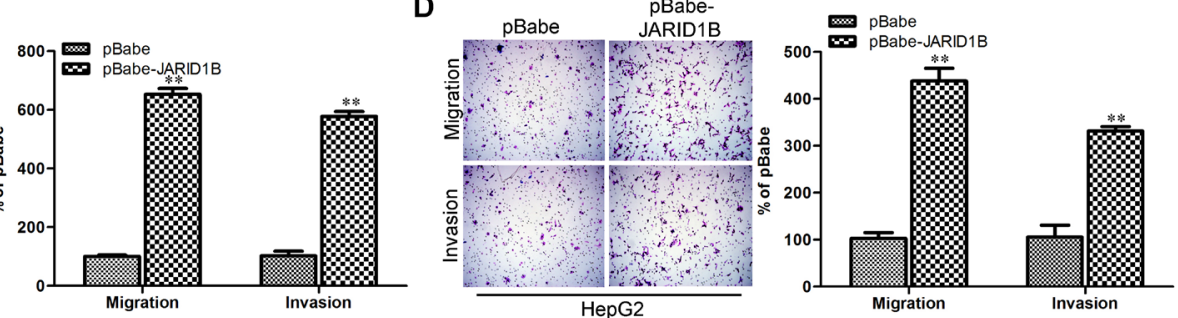

$\mathbf{F}$
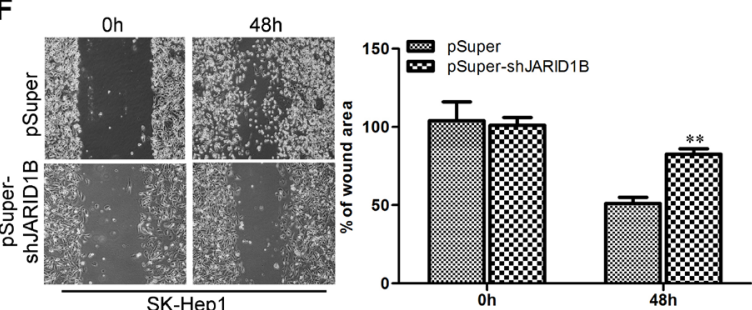

H
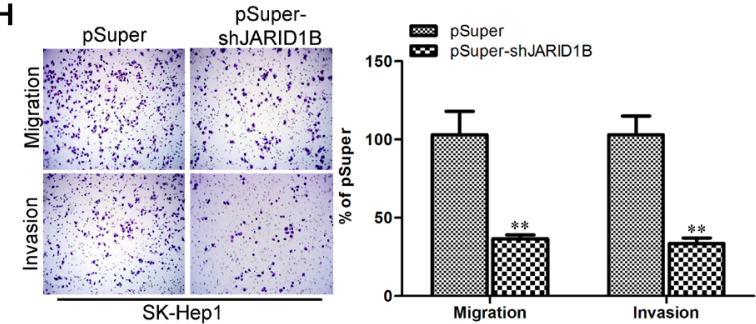

Figure 5: JARID1B promotes migratory and invasive capacities of HCC cells in vitro. Huh7-pBabe-JARID1B (A), HepG2pBabe-JARID1B (B) and theirs control vector cells were subjected to wound healing assays; the uncovered areas in the wound healing assays were quantified as a percentage of the original wound area. Huh7-pBabe-JARID1B (C), HepG2-pBabe-JARID1B (D) and theirs control vector cells were subjected to Transwell migration (C and D, top), and Matrigel invasion assays (C and D, bottom), quantification of migrated cells through the membrane and invaded cells through Matrigel of each cell line are shown as proportions of their vector controls. SNU423-pSuper-shJARID1B (E), SK-Hep1-pSuper-shJARID1B (F) and theirs control vector cells were subjected to wound healing assays, the uncovered areas in the wound healing assays were quantified as a percentage of the original wound area. SNU423pSuper-shJARID1B (G), SK-Hep1-pSuper-shJARID1B (H) and theirs control vector cells were subjected to Transwell migration (G and $\mathrm{H}$, top), and Matrigel invasion assays ( $\mathrm{G}$ and $\mathrm{H}$, bottom), quantification of migrated cells through the membrane and invaded cells through Matrigel of each cell line are shown as proportions of their vector controls. **, $P<0.01$ is based on the Student $t$ test. All results are from three independent experiments. Error bars, SD. 


\section{JARID1B promotes tumorigenesis and metastasis in vivo}

To extend our in vitro observations, we investigated whether JARID1B could regulate tumorigenic and metastatic capacity of HCC cells in vivo. HepG2-pBabeJARID1B， SK-Hep1-pSuper-shJARID1B and their corresponding control cells were subcutaneously injected into nude mice. Tumor size was measured every week up to 6 weeks. As expected, the tumors from HepG2-pBabeJARID1B cells grew more rapidly at the implantation site than the control cells (Figure 6A - C). In contrast, silencing JARID1B in the typically aggressive SK-Hep1 cells led to a dramatic decrease in tumor volume and weight (Figure $6 \mathrm{D}-\mathrm{F})$.

We then investigated the functional relevance of JARID1B for metastasis in vivo. HepG2-pBabe-JARID1B, SK-Hep1-pSuper-shJARID1B and their corresponding
A

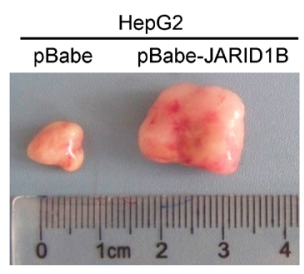

D

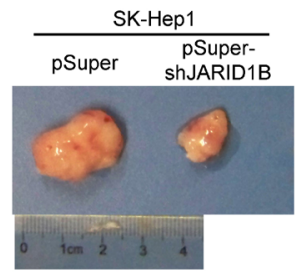

B

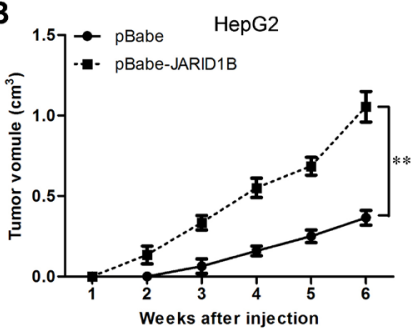

E

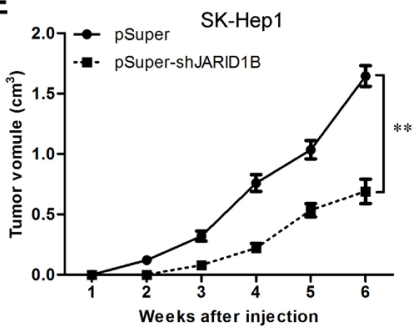

C

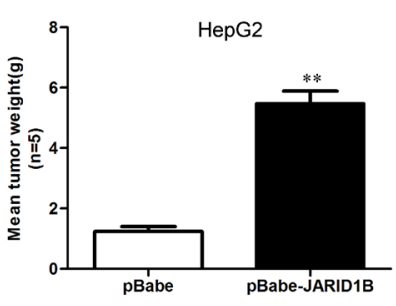

F

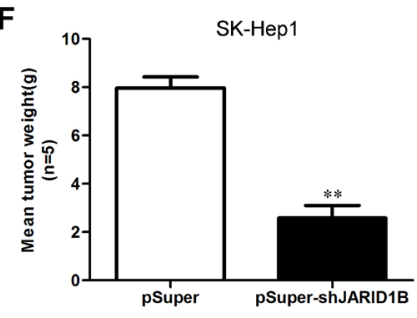

G

\begin{tabular}{lc}
\hline & $\begin{array}{c}\text { Number of mice with } \\
\text { lung metastasis }\end{array}$ \\
\hline HepG2 pBAbe & $2 / 5$ \\
HepG2 pBabe-JARID1B & $5 / 5$ \\
SK-Hep1 pSuper & $5 / 5$ \\
SK-Hep1 pSuper-shJARID1B & $0 / 5$ \\
\hline
\end{tabular}

I

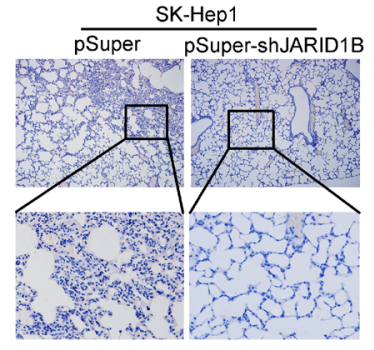

$\mathbf{H}$
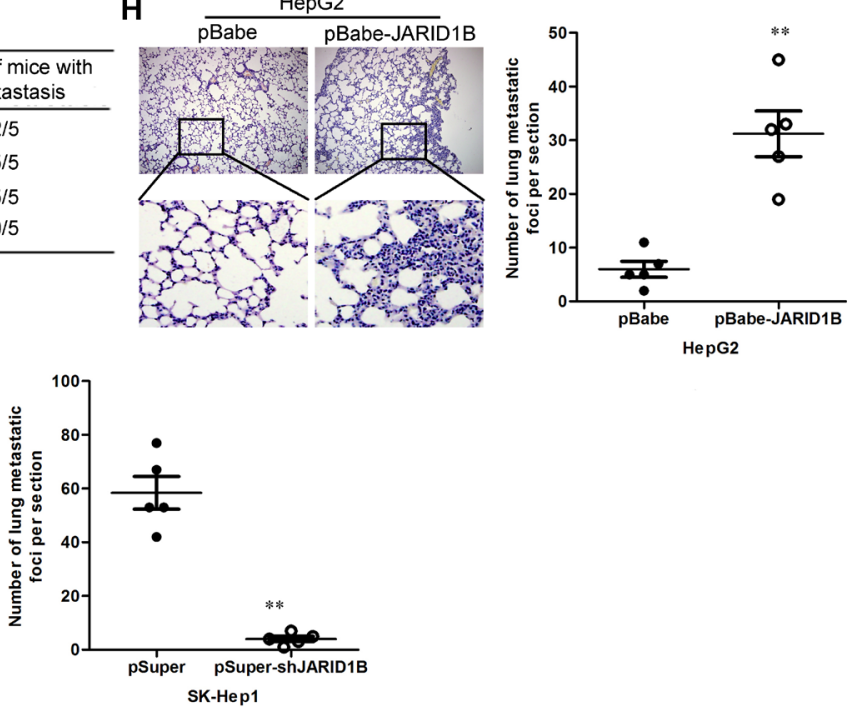

Figure 6: JARID1B promotes tumorigenesis and metastasis in vivo. A and $\mathbf{D}$, representative images of HepG2-pBabe-JARID1B (A), SK-Hep1-pSuper-shJARID1B (D) or their control cell tumors by subcutaneous injection. $\mathbf{B}$ and $\mathbf{E}$, growth curve of tumors formed by HepG2-pBabe-JARID1B (B), SK-Hep1-pSuper-shJARID1B (E) or their control cells by subcutaneous injection. C and F, the weight of tumors formed by HepG2-pBabe-JARID1B (C), SK-Hep1-pSuper-shJARID1B (F) or their control cells at harvest time. G, the total numbers of mice with distant metastasis at 60 days after injection of HepG2-pBabe-JARID1B, SK-Hep1-pSuper-shJARID1B or their control cells. $\mathbf{H}$ and $\mathbf{I}$, the numbers of metastatic foci per section in lung of individual mouse with injection of HepG2-pBabe-JARID1B (H), SK-Hep1-pSuper-shJARID1B (I) or their control cells. $\mathrm{n}=5$ for subcutaneous transplantation and $\mathrm{n}=5$ for tail vein injection. $* *, P<$ 0.01 is based on the Student $t$ test. All results are from three independent experiments. Error bars, SD. 
control cells were injected into nude mice through the tail vein. JARID1B overexpression not only significantly increased the number of mice with distant metastasis (Figure 6G), but also dramatically increased the number of metastatic tumors in lung of each mouse (Figure $6 \mathrm{H}$ ). Silencing JARID1B in SK-Hep1 cells inhibited metastatic behavior, both in terms of the number of mice with distant metastasis (Figure 6G) and the number of metastatic tumors in the lung of each mouse (Figure 6I). Therefore, the in vivo results further demonstrate the critical role of JARID1B in HCC metastasis.

\section{JARID1B regulates PTEN expression through H3K4 trimethylation}

To better understand the mechanisms by which JARID1B engaged in HCC development and progression, we performed gene expression profiling on HepG2-pBabeJARID1B and its control cells. Microarray analyses identified a list of genes significantly differentially expressed after JARID1B overexpression including downregulation of PTEN (Figure 7A). Furthermore, gene set enrichment analysis indicated that proliferation, neoplasm metastasis and invasion, cell movement and motility, and PTEN related gene signatures were significantly changed in JARID1B overexpression cells (Figure 7B), supporting the idea that JARID1B regulates proliferation, EMT and cancer invasion and metastasis. These data also led us to hypothesize that JARID1B exerts these functions possibly via PTEN. To test this, we first determined whether PTEN is a downstream target of JARID1B in HCC cells. Expression of PTEN in the cells with altered JARID1B expression was further evaluated by qRT-PCR and Western blotting. Huh7-pBabeJARID1B and HepG2-pBabe-JARID1B cells exhibited greatly increased both PTEN mRNA and protein levels (Supplemental Figure 4A, Figure 7C), whereas silencing JARID1B in SNU423 and SK-Hep1 cells dramatically decreased its mRNA and protein levels (Supplemental Figure 4B, Figure 7D). Suggesting the regulation of PTEN expression by JARID1B is at transcriptional level. It has been shown that PTEN can regulate PI3K/Akt pathway in many tumor cells $[24,25]$. So we next examined the role of JARID1B-mediated inhibition of PTEN in the PI3K/ Akt pathway. Of note, ectopic JARID1B expression in Huh7 and HepG2 cells remarkably increased the level of phosphorylated PI3K and AKT (Figure 7C), whereas silencing JARID1B in SNU423 and SK-Hep1 cells robustly suppressed phosphorylation of PI3K and AKT (Figure 7D). While both ectopic expression and silencing of JARID1B not altered the expression of PI3K and AKT mRNA levels (Supplemental Figure 4A and 4B).

We then explored how JARID1B regulates PTEN expression at the transcriptional level. JARID1B complexes are frequently involved in chromatin regulation
$[26,27]$. To determine whether JARID1B regulates specific histone modifications in HCC cells, histone modification patterns were measured after modulation of JARID1B expression. Among histone H3K4 and H3K27, we found that only H3K4 was affected by JARID1B. Ectopic expression of JARID1B decreased H3K4me1, $\mathrm{H} 3 \mathrm{~K} 4 \mathrm{me}$, and H3K4me3 (Figure 7E) while silencing of JARID1B increased these modification (Supplemental Figure $4 \mathrm{C}$ ). Because $\mathrm{H} 3 \mathrm{~K} 4 \mathrm{me} 3$ is associated with active transcription, we tested whether JARID1B expression was correlated with the $\mathrm{H} 3 \mathrm{~K} 4 \mathrm{me} 3$ modification at the PTEN gene promoter in HCC cells. Quantitative chromatin immunoprecipitation (qChIP) assays were performed in HepG2-pBabe-JARID1B, SK-Hep1-pSuper-shJARID1B and their corresponding control cells. We found that JARID1B expression was associated with decreased H3K4me3 levels at region -1423 to -1176bp (Figure 7F1) and -741 to $-513 \mathrm{bp}$ (Figure 7F2) of the PTEN promoter in HepG2-pBabe-JARID1B cells (Figure 7G). More occupancy of those PTEN gene promoter regions by H3K4me3 was detected in SK-Hep1-pSuper-shJARID1B cells (Figure $7 \mathrm{H}$ ). These results clearly indicate that JARID1B induces transcriptional inactivation of PTEN through regulating $\mathrm{H} 3 \mathrm{~K} 4 \mathrm{me} 3$ and decreasing $\mathrm{H} 3 \mathrm{~K} 4 \mathrm{me} 3$ to the PTEN gene promoter.

\section{PTEN is a mediator for JARID1B-induced EMT, migration, invasion, and tumor metastasis in HCC cells}

On the basis of the indispensable role of PTEN in the biologic functions of JARID1B, we overexpressed PTEN in HepG2-pBabe-JARID1B cells (Figure 8A) and silenced PTEN in SK-Hep1-pSuper-shJARID1B cells (Figure 8E). Of note, PTEN overexpression significantly decreased phosphorylation of P13K and Akt in HepG2pBabe-JARID1B cells (Figure 8B). Moreover PTEN overexpression significantly decreased the proliferation (Figure 8C) as well as migration, invasion of HepG2pBabe-JARID1B cells (Figure 8D), and EMT markers changes (Supplemental Figure 6C). Meanwhile silencing PTEN increased phosphorylation of P13K and AKT in SK-Hep1-pSuper-shJARID1B cells (Figure 8F). Moreover silencing PTEN in SK-Hep1-pSuper-shJARID1B cells significantly increased the proliferation (Figure 8G) as well as migration, invasion (Figure 8H), and EMT markers changes (Supplemental Figure 6D). Taken together, these results show that PTEN mediates JARID1B-induced EMT, migration and invasion in hepatocellular carcinoma cells.

We next tested the effect of specific blockage of AKT and PI3K pathways on the proliferative and migrative functions of JARID1B. As shown in Supplemental Figure 5A and Supplemental Figure 5D, treatment with a specific PI3K inhibitor, LY294002, or an AKT inhibitor drastically retarded the level of phosphorylated PI3K and AKT of 
A

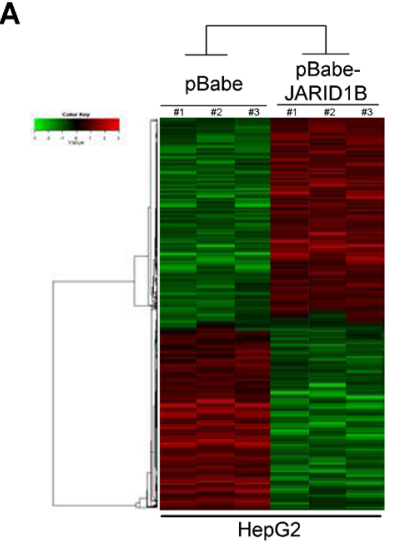

C

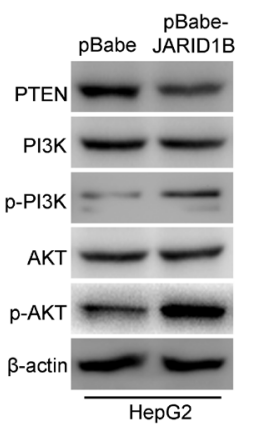

B
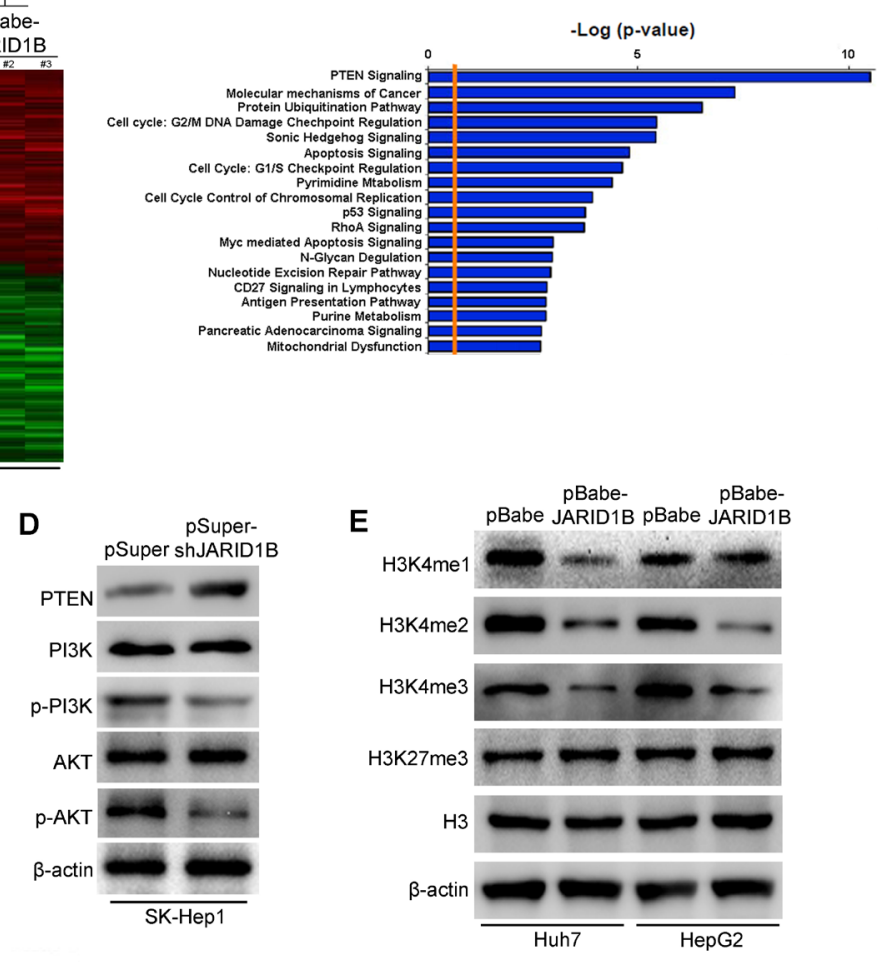

$\mathbf{F}$

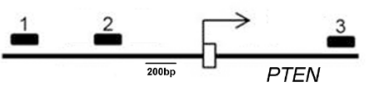

G
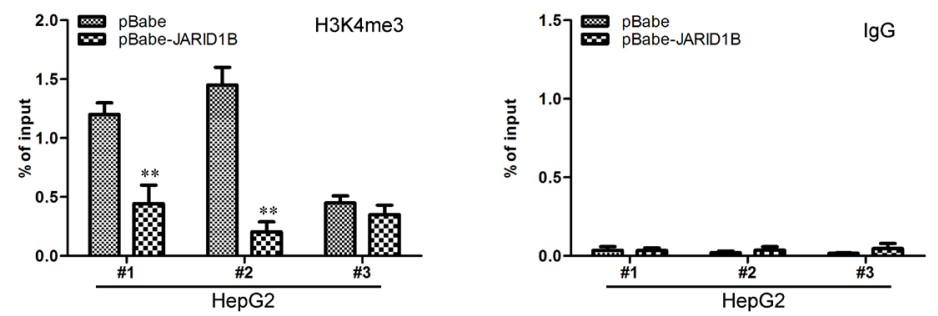

H
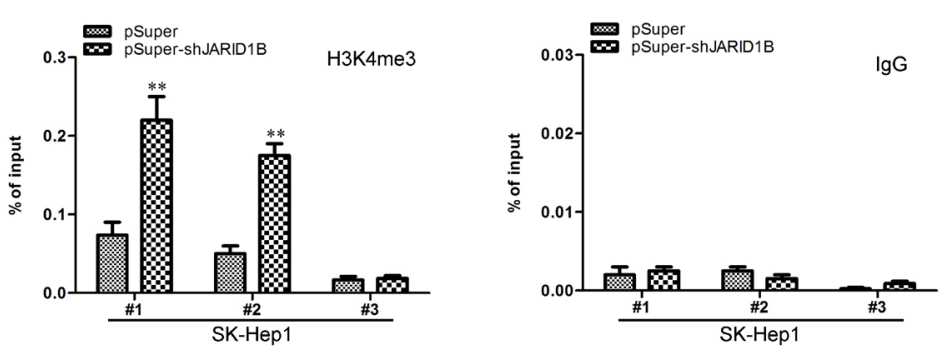

Figure 7: JARID1B downregulates PTEN expression in HCC cells. A, supervised hierarchical clustering of the genes differentially expressed after JARID1B overexpression in HepG2 cells. B, gene set enrichment analysis was carried out using ConceptGen. $\mathbf{C}$ and $\mathbf{D}$, protein levels of PTEN, PI3K, p-PI3K, AKT, and p-AKT were measured in HCC cells with JARID1B overexpression (C) or silencing (D) by Western blot assay. E, the abundance of $\mathrm{H} 3$ lysine methylation was assessed in HCC cells with JARID1B overexpression by Western blotting using whole-cell lysate; total $\mathrm{H} 3$ and $\beta$-actin were used as a loading control. F, schematic presentation of three regions relative to the PTEN transcriptional start site used as primers to test histone occupied abundance. $\mathbf{G}$ and $\mathbf{H}$, qChIP was performed to assess H3K4me3 occupancy in HepG2-pBabe-JARID1B (G), SK-Hep1-pSuper-shJARID1B (H) or their control cells. IgG was used as negative control ( $\mathrm{G}$ and $\mathrm{H}$, left). "Percentage of input" indicates the ratio of DNA fragment of each promoter region bound by $\mathrm{H} 3 \mathrm{~K} 4 \mathrm{me} 3$ to the total amount of input DNA fragment without $\mathrm{H} 3 \mathrm{~K} 4 \mathrm{me} 3$ antibody pull-down. ${ }^{*}, P<0.01$ is based on the Student $t$ test. All results are from three independent experiments. Error bars, SD. 
A

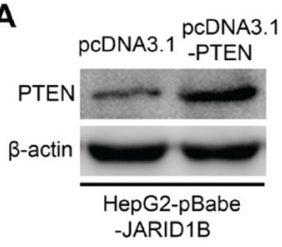

B

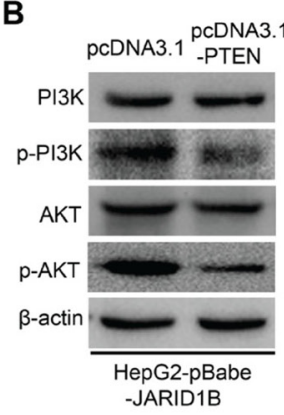

C

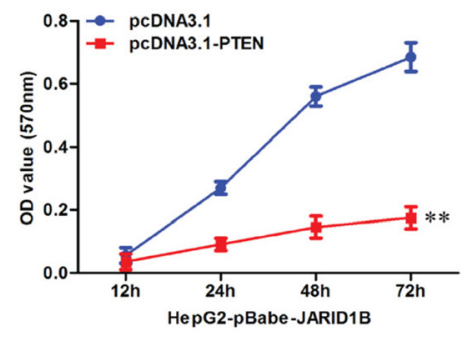

$\mathbf{E}$

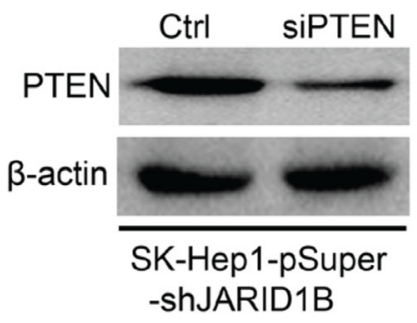

$\mathbf{F}$

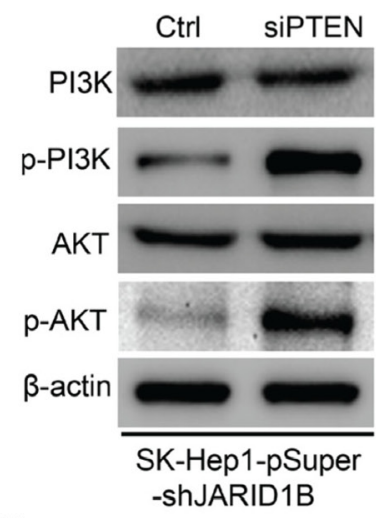

$\mathrm{H}$

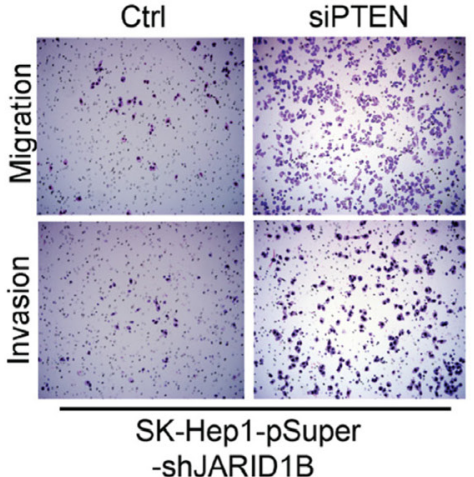

G
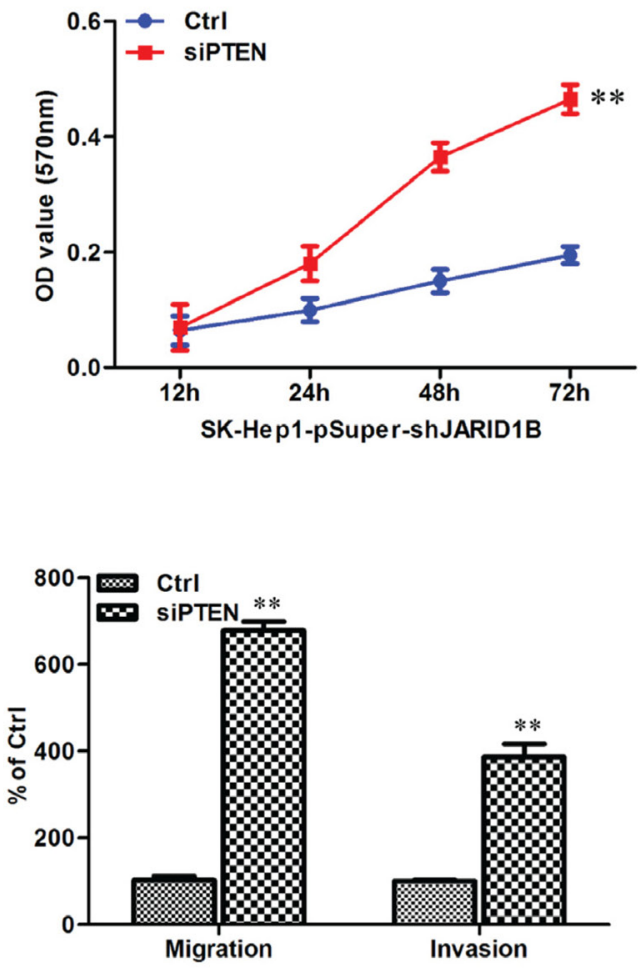

Figure 8: JARID1B regulates PTEN transcriptional expression through H3K4 trimethylation. A and $B$, the abundance of H3 lysine methylation was assessed in HCC cells with JARID1B overexpression (A) or silencing (B) by Western blotting using whole-cell lysate; total $\mathrm{H} 3$ and $\beta$-actin were used as a loading control. $\mathbf{C}$, schematic presentation of three regions relative to the PTEN transcriptional start site used as primers to test histone occupied abundance. D and E, qChIP was performed to assess H3K4me3 occupancy in HepG2pBabe-JARID1B (D), SK-Hep1-pSuper-shJARID1B (E) or their control cells. IgG was used as negative control (D and E, left). "Percentage of input" indicates the ratio of DNA fragment of each promoter region bound by H3K4me3 to the total amount of input DNA fragment without $\mathrm{H} 3 \mathrm{~K} 4 \mathrm{me} 3$ antibody pull-down. ${ }^{* *}, P<0.01$ is based on the Student $t$ test. All results are from three independent experiments. Error bars, SD. 
JARID1B-overexpressing HepG2 cells, and considerably weakened the abilities of these JARID1B-transduced cells to induce proliferation, migration, invasion and EMT markers changes (Supplemental Figure 5B and 5C, Supplemental Figure 5E and 5F, Supplemental Figure 6A and $6 \mathrm{~B})$. These data suggested that JARID1B promoted the proliferative and EMT of HCC cells by simultaneously activating both PI3K and AKT pathways.

\section{DISCUSSION}

Hepatocellular carcinoma (HCC) belongs to common and aggressive human malignancies and is one of the leading causes of death by tumor worldwide [1]. The high mortality rate of $\mathrm{HCC}$ is caused by frequent tumor metastasis, postsurgical recurrence, and late detection at advanced stages [1]. However, good diagnostic markers, drug targets and therapeutic strategies are still insufficient for successful treatment of HCC [1]. Histone methylation is a dynamic process that participates in a diverse array of cellular processes and has been found to associate with cancer, including HCC [28, 29]. Recently, several histone demethylases have been identified that catalyze the removal of methylation from histone $\mathrm{H} 3$ lysine residues $[30,31]$. Previous studies had found that JARID1B, also named Plu-1, as a histone demethylase that can catalyze the removal of all three methyl groups from the H3K4 lysine residue [4]. However, its role and underlying mechanisms in HCCs still unknown.

To our knowledge, this is the first research to show that JARID1B plays an important part in HCC. We found that as compared with normal tissues, $\mathrm{HCC}$ specimens showed overexpression of JARID1B and its overexpression was significantly correlated with distant metastasis in HCC tissues. High expression of JARID1B was associated with decreased overall survival of HCC patients. JARID1B overexpression in HCC cells induced proliferation, EMT, migration, invasion in vitro and enhanced tumorigenic and metastatic capacities in vivo. In contrast, silencing JARID1B reversed these events in invasive $\mathrm{HCC}$ cells. We also showed that a mechanistic link between JARID1B and PTEN through JARID1Bmediated regulation of $\mathrm{H} 3 \mathrm{~K} 4 \mathrm{~m} 3$, which subsequently leads to transcriptional downregulation of PTEN expression. Overexpression of PTEN attenuated JARID1B function and had effects similar to those elicited by direct silencing of JARID1B. These results lead us to propose a model for JARID1B regulation of EMT and metastasis through transcriptional regulation of PTEN in HCCs.

The putative role of JARID1B as an oncogene in cancer development is supported by the observations that JARID1B is highly expressed in prostate cancer and other malignant tumors relative to normal tissues $[12,23,32$, 13]. The fact that JARID1B as a demethylase capable of removing three methyl groups from histone $\mathrm{H} 3$ lysine 4, subsequently downregulation several well-known tumor suppressor genes [27, 33, 34]. Perhaps most convincing is functional evidence showing that the JARID1B demethylase contributes to tumor cell proliferation through the epigenetic repression of a tumor suppressor let-7e in breast cancer cells [8]. Consistent with this research, in the present study we showed that overexpression of JARID1B promoted HCC cells proliferation and enhanced tumor formation in vivo. Interestingly, our study points to a novel function of JARID1B in HCC metastasis through regulating EMT.

HCC cells with ectopic expression of JARID1B displayed an EMT phenotype, including the associated stimulatory effects on migration and invasion in vitro. Interestingly, our results indicate that JARID1B not only promotes EMT, but silencing of JARID1B also leads to MET. All of these characteristics induced by JARID1B in vitro culminated to increased numbers of distant metastases in vivo. These empirical findings provide a mechanistic framework to explain the clinical observations that HCC patients with high levels of JARID1B in tissue samples have more chance of distant metastasis, a significantly shorter overall and disease-free survival.

The roles of several transcription factors as EMT regulators have been extensively reported [16, 35]. In our effort to elucidate the mechanism how JARID1B modulates EMT in HCC cells, we identified PTEN as an effective mediator of JARID1B-induced these phenomena. PTEN is one of the most frequently mutated tumor suppressors in human cancer including HCCs [36, 24]. PTEN also suppresses migration, genetic deletion of the PTEN tumor suppressor gene promotes cell motility, and PTEN reconstitution or overexpression inhibits cell motility in a variety of cell types. Mechanistically, PTEN reduces cell motility through a variety of pathways, and $\mathrm{PI} 3 \mathrm{~K} / \mathrm{AKT}$ is one important target of PTEN $[37,38]$. The mechanistic connection between JARID1B and PTEN was previously unknown. In this study, we showed that modulation of JARID1B expression altered the methylation status of $\mathrm{H} 3 \mathrm{~K} 4$ at the PTEN gene promoter, which in turn transcriptionally controlled expression of PTEN. However, we did not detect any influence of JARID1B expression on the methylation status of H3K27. Thus, we conclude that JARID1B transcriptionally inactivates PTEN expression through $\mathrm{H} 3 \mathrm{~K} 4$ demethylation and reduces $\mathrm{H} 3 \mathrm{~K} 4 \mathrm{me} 3$ to the PTEN gene promoter, and consequently inhibit phosphorylated PI3K and AKT, resulting in increase in proliferation, migration and invasion both in vitro and in vivo.

EMT is essential for tumor cells to disseminate from adjacent tissues and seed new tumors in distant sites [15]. Our results demonstrated that JARID1B regulated EMT in addition to proliferation, and JARID1B-induced processes are reversible with the suppression of JARID1B expression, providing an optimal therapeutic option to manipulate JARID1B levels in HCC patients' clinical practice. 


\section{MATERIALS AND METHODS}

\section{Chemicals and antibodies}

Lipofectamine 2000 transfection and TRIZOL LS Reagents were purchased from Invitrogen (Grand Island, NY, USA). Antibodies against JARID1B, Histone H3, H3K4me1 (monomethylated K4), H3K4me2 (dimethylated K4), H3K4me3 (trimethylated K4), H3K27me3 (trimethylated K27) were purchased from Abcam (Cambridge, MA, USA). E-cadherin, N-cadherin, vimentin, PTEN, cyclin D1 and $\beta$-actin antibodies were from Cell Signaling technology (Danvers, MA, USA). Anti- $\alpha$-catenin antibody was from BD (Franklin Lakes, NJ, USA). Unless otherwise noted, all other chemicals were from Sigma (St. Louis, MO, USA).

\section{Patients and specimens}

Thirty-eight tumor and para-cancerous tissues which, were used for qRT-PCR and Western blot analysis, were randomly collected from HCC patients who underwent curative resection with informed consent between 2011 and 2013 at the Department of Hepatobiliary Surgery, Guilin Medical University, Affiliated Hospital, Guilin, China. All tissues were collected immediately upon resection of the tumors in the operation theater, transported in liquid nitrogen, and then stored at $-80^{\circ} \mathrm{C}$. Another 178 hepatocellular carcinoma tissues which, were used for immunohistochemical analysis, were randomly collected from HCC patients who underwent curative resection with informed consent between 2006 and 2009 at the Department of Hepatobiliary Surgery, Guilin Medical University, Affiliated Hospital, Guilin, China and Department of General Surgery, The First Affiliated Hospital, Sun Yat-Sen University, Guangzhou, China. Tumor staging was based on the 6th edition of the tumor-node-metastasis (TNM) classification of the International Union Against Cancer. The clinicopathologic characteristics of the 178 hepatocellular carcinoma tissues are summarized in Table 1. Follow-up data were summarized at the end of October 2014, with a median observation time of 65.3 months. Study protocols were approved by the Hospital Ethics Committee of Guilin Medical University, and written informed consent was obtained from patients based on the Declaration of Helsinki.

\section{Histological and immunohistochemical analysis}

The normal human liver tissues, human tumor tissues, and lungs dissected from mice were fixed in $4 \%$ paraformaldehyde in phosphate-buffered saline
(PBS) overnight and subsequently embedded in paraffin wax. Sections cut at a thickness of $4 \mu \mathrm{m}$ were stained with hematoxylin and eosin for histological analysis. Immunohistochemical analysis was performed for different markers in these arrays as described previously [18].The proportion of stained cells (lower, $<30 \%$ staining; higher, $\geq 30 \%$ staining) was semiquantitatively determined following published protocols [19].

\section{Cell culture}

HCC cells (ATCC, Manassas, VA, USA) were cultured under the following conditions: SNU-423, SNU449 , and SNU-886 cell lines were cultured using $10 \%$ fetal bovine serum (Cat\#10099-141, Invitrogen, Carlsbad, CA) in either RPMI-1640 (Cat\#C11875, Invitrogen). Huh7, HepG2, and SK-Hep-1 cell lines were cultured using 10\% fetal bovine serum (Invitrogen) in Dulbecco's modified Eagle medium (Cat\#C11965, Invitrogen). Cell culture was according to manufacturer's protocol. All the cell lines were grown at $37^{\circ} \mathrm{C}$ in a $5 \% \mathrm{CO}_{2} / 95 \%$ air atmosphere and were revived every 3 to 4 months.

\section{Establishment of JARID1B and PTEN stable expression and JARID1B and PTEN knockdown cell lines}

Retroviral construct containing human pBabeJARIDIB cDNA, pcDNA3.1-PTEN cDNA and pSuper. retro.puro with shRNA against human JARIDIB and siPTEN were prepared as described previously [20]. The generation of retrovirus supernatants and transfection of hepatocellular carcinoma cells were conducted as described previously [20]. The expression of JARID1B and PTEN was confirmed by qRT-PCR and Western blotting analysis.

\section{Cell proliferation assay}

Cells were seeded in 96-well plates in triplicate at densities of $1 \times 10^{3}$ per well. Cell proliferation was monitored at desired time points using 3-(4, 5-dimethylthiazol-2-yl)-2, 5-diphenyltetrazolium bromide (MTT). In brief, the MTT assay was performed by adding $20 \mu \mathrm{l}$ MTT $(5 \mathrm{mg} / \mathrm{ml})$ for $4 \mathrm{~h}$. Light absorbance of the solution was measured at $570 \mathrm{~nm}$ on a microplate reader.

\section{Colony formation assay}

Cells were seeded in triplicate at 500 cells $/ 6-\mathrm{cm}$ dishes in complete medium. After 3 weeks of growth, the cells were fixed and stained with crystal violet $(0.1 \%$, w $/ \mathrm{v}$ in 20nM 4-morpholinepropanesulfonic acid), and visible 
colonies were counted according to cell numbers in each colony.

\section{Wound healing assay}

Cells were seeded in $6 \mathrm{~cm}$ culture plates, and the cell monolayers were wounded by scratching with sterile plastic $200 \mu \mathrm{l}$ micropipette tips and photographed using phase-contrast microscopy. The migration distance of each cell was measured after the photographs were converted to Photoshop files.

\section{Cell invasion and motility assay}

Invasion of cells was measured in Matrigel (BD, Franklin Lakes, NJ, USA) -coated Transwell inserts (6.5mm, Costar, Manassas, VA, USA) containing polycarbonate filters with $8-\mu \mathrm{m}$ pores as detailed previously [21]. The inserts were coated with $50 \mu \mathrm{l}$ of $1 \mathrm{mg} / \mathrm{ml}$ Matrigel matrix according to the manufacturer's recommendations. $2 \times 10^{5}$ cells in $200 \mu \mathrm{l}$ of serum-free medium were plated in the upper chamber, whereas $600 \mu \mathrm{l}$ of medium with $10 \%$ fatal bovine serum were added to lower well. After $24 \mathrm{hrs}$ incubation, cells that migrated to the lower surface of the membrane were fixed and stained. For each membrane, five random fields were counted at $\times 10$ magnification. Motility assays were similar to Matrigel invasion assay except that the Transwell insert was not coated with Matrigel.

\section{Confocal immunofluorescence microscopy}

Cell lines were plated on culture slides (Costar, Manassas, VA, USA). After 24hrs, the cells were rinsed with PBS and fixed with 4\% paraformaldehyde, and cell membrane was permeabilized using $0.5 \%$ Triton X-100. These cells were then blocked for $30 \mathrm{~min}$ in $10 \% \mathrm{BSA}$ and then incubated with primary antibodies overnight at $4^{\circ} \mathrm{C}$. After three washes in PBS, the slides were incubated for 1 hour in the dark with FITC-conjugated secondary antibodies (Invitrogen, Grand Island, NY, USA). After three further washes, the slides were stained with DAPI for $5 \mathrm{~min}$ to visualize the nuclei, and examined using a Carl Zeiss confocal imaging system (LSM 780) (Carl Zeiss, Jena, Germany).

\section{Western blotting}

Cells were lysed in lysis buffer and total protein contents were determined by the Bradford method. $30 \mu \mathrm{g}$ of lysis were separated by reducing SDS-PAGE and probed with specific antibodies. Blots were washed and probed with respective secondary peroxidaseconjugated antibodies, and the bands visualized by chemoluminescence (Amersham Biosciences).

\section{qRT-PCR}

Total RNA was extracted using Trizol reagent and cDNA was synthesized using SuperScript II Reverse Transcriptase (Invitrogen). qRT-PCR and data collection were performed with an ABI PRISM 7900HT sequence detection system. The primers used for the amplification of the indicated genes are available upon request.

\section{Gene expression profiling}

Total RNA quality and quantity were determined using Agilent 2100 Bioanalyzer and NanoDrop ND1000. Affymetrix HU U133 plus 2.0 arrays were used according to manufacturer's protocol. The data were initially normalized by robust multiarray average (RMA) normalization algorithms in expression console software (Affymetrix). Significantly altered genes between JARID1B overexpression and its control cells were considered by scatter plots and the genes up- and downregulated $\geq 5$-fold. Clustering analysis was done using gene list by Gene Cluster v3.0 software, and heat maps were visualized using Java TreeView v1.1.4r3 software. Gene set enrichment analysis was carried out using ConceptGen (http://conceptgen.ncibi.org/core/conceptGen/index.jsp). Gene sets were either obtained from the ConceptGen or from published gene signatures.

\section{Chromatin immunoprecipitation (ChIP)-qPCR}

Chromatin Immunoprecipitation kit (Cat. 17-371) was purchased from Millipore and ChIP experiments were carried out essentially as described [22]. Immnuoprecipitated DNA was analyzed on the ABI PRISM 7900HT sequence detection system. The primers used for detection of promoters after ChIP are available upon request.

\section{In vivo tumor growth and metastasis}

Nude mice were purchased from the Shanghai Slac Laboratory Animal Co. Ltd and maintained in microisolator cages. All animals were used in accordance with institutional guidelines and the current experiments were approved by the Use Committee for Animal Care. For subcutaneous inoculation, different numbers of tumor cells were resuspended in PBS medium with 50\% Matrigel and inoculated subcutaneously into the 8-week-old nude mice. The tumors were measured weekly and the tumor volume was calculated according to the formula length $\times \operatorname{width}^{2} / 2$. The mice were killed six weeks after the inoculation. For metastasis assays, cells were resuspended in PBS at a 
concentration of $1 \times 10^{7}$ cells $\mathrm{ml}^{-1}$. Cell suspension $(0.1 \mathrm{ml})$ was injected into tail veins of nude mice. All of the mice were killed by $\mathrm{CO}_{2} 60$ days after inoculation.

\section{Statistical analysis}

Results were analyzed with SPSS13.0 statistical software. Correlation between JARID1B expression and clinicopathologic parameters was evaluated using the Chisquare $(\chi 2)$ test, and quantitative variables were analyzed by the independent $t$ test. The survival probability was estimated by Kaplan-Meier method, and the comparison of survival curves between groups was done with the logrank test. The statistical significance of the differences between mean values was determined by $P<0.05$.

\section{ACKNOWLEDGMENTS}

This research was supported in part by The National Natural Science Foundation of China (No.81060094, No. 81360367, No. 81160066 and No. 30870719); Scientific Research Foundation for Returned Scholars, Ministry of Education of China (jyb2010-01); Major Project of Science Research of Guangxi Universities (2013ZD046); The Natural Science Foundation of Guangxi (2014GXNSFBA118162), Special Project of Traditional Chinese Medicine of Guangxi Health Department (GZPT13-45), Guangxi Distinguished Experts Special Fund, Project supported by the Guangxi culture of new century academic and technical leader of special funds.

\section{DISCLOSURE OF POTENTIAL CONFLICTS OF INTEREST}

No potential conflicts of interest were disclosed by all authors.

\section{REFERENCES}

1. Forner A, Llovet JM and Bruix J. Hepatocellular carcinoma. Lancet. 2012; 379:1245-55.

2. Santoro A, Rimassa L, Borbath I, Daniele B, Salvagni S, Van Laethem JL, Van Vlierberghe H, Trojan J, Kolligs FT, Weiss A, Miles S, Gasbarrini A, Lencioni M et al. Tivantinib for second-line treatment of advanced hepatocellular carcinoma: a randomised, placebo-controlled phase 2 study. Lancet Oncol. 2013; 14:55-63.

3. Baek KK, Kim JH, Uhm JE, Park SH, Lee J, Park JO, Park YS, Kang WK and Lim HY. Prognostic factors in patients with advanced hepatocellular carcinoma treated with sorafenib: a retrospective comparison with previously known prognostic models. Oncology. 2011; 80:167-74.

4. Xiang Y, Zhu Z, Han G, Ye X, Xu B, Peng Z, Ma Y, Yu Y, Lin H, Chen AP and Chen CD. JARID1B is a histone H3 lysine 4 demethylase up-regulated in prostate cancer. Proc Natl Acad Sci U S A. 2007; 104:19226-31.

5. Scibetta AG, Santangelo S, Coleman J, Hall D, Chaplin T, Copier J, Catchpole S, Burchell J and Taylor-Papadimitriou J. Functional analysis of the transcription repressor PLU-1/ JARID1B. Mol Cell Biol. 2007; 27:7220-35.

6. Benevolenskaya EV. Histone H3K4 demethylases are essential in development and differentiation. Biochem Cell Biol. 2007; 85:435-43.

7. Catchpole S, Spencer-Dene B, Hall D, Santangelo S, Rosewell I, Guenatri M, Beatson R, Scibetta AG, Burchell JM and Taylor-Papadimitriou J. PLU-1/JARID1B/KDM5B is required for embryonic survival and contributes to cell proliferation in the mammary gland and in ER+ breast cancer cells. Int J Oncol. 2011; 381267-77.

8. Mitra D, Das PM, Huynh FC and Jones FE. Jumonji/ARID1 B (JARID1B) protein promotes breast tumor cell cycle progression through epigenetic repression of microRNA let-7e. J Biol Chem. 2011; 286:40531-5.

9. Zeng J, Ge Z, Wang L, Li Q, Wang N, Bjorkholm M, Jia J and $\mathrm{Xu}$ D. The histone demethylase RBP2 Is overexpressed in gastric cancer and its inhibition triggers senescence of cancer cells. Gastroenterology. 2010; 138:981-92.

10. Zhou M, Zeng J, Wang X, Huang T, Fu Y, Sun T, Jia J and Chen C. Histone demethylase RBP2 decreases miR-21 in blast crisis of chronic myeloid leukemia. Oncotarget. 2015; 6:1249-61

11. Liang $X$, Zeng J, Wang L, Fang $M$, Wang Q, Zhao M, Xu X, Liu Z, Li W, Liu S, Yu H, Jia J and Chen C. Histone demethylase retinoblastoma binding protein 2 is overexpressed in hepatocellular carcinoma and negatively regulated by hsa-miR-212. PLoS One. 2013; 8(7).

12. Yamamoto S, Wu Z, Russnes HG, Takagi S, Peluffo G, Vaske C, Zhao X, Moen Vollan HK, Maruyama R, Ekram MB, Sun H, Kim JH, Carver K et al. JARID1B is a luminal lineage-driving oncogene in breast cancer. Cancer Cell. 2014; 25:762-77.

13. Kano Y, Konno M, Ohta K, Haraguchi N, Nishikawa S, Kagawa Y, Hamabe A, Hasegawa S, Ogawa H, Fukusumi T, Noguchi Y, Ozaki M, Kudo T et al. Jumonji/Arid1b (Jarid1b) protein modulates human esophageal cancer cell growth. Mol Clin Oncol. 2013; 1:753-7.

14. Zurkiya $\mathrm{O}$ and Ganguli S. Beyond hepatocellular carcinoma and colorectal metastasis: the expanding applications of radioembolization. Front Oncol. 2014; 4(150).

15. Maheswaran T and Rushbrook SM. Epithelial-mesenchymal transition and the liver: role in hepatocellular carcinoma and liver fibrosis. J Gastroenterol Hepatol. 2012; 27:418-20.

16. Li YM, Xu SC, Li J, Han KQ, Pi HF, Zheng L, Zuo GH, Huang XB, Li HY, Zhao HZ, Yu ZP, Zhou Z and Liang $P$. Epithelial-mesenchymal transition markers expressed in circulating tumor cells in hepatocellular carcinoma patients with different stages of disease. Cell Death Dis. 2013; $4: \mathrm{e} 831$. 
17. Panebianco C, Saracino C and Pazienza V. Epithelialmesenchymal transition: molecular pathways of hepatitis viruses-induced hepatocellular carcinoma progression. Tumour Biol. 2014; 35:7307-15.

18. Liu WR, Tian MX, Yang LX, Lin YL, Jin L, Ding ZB, Shen YH, Peng YF, Gao DM, Zhou J, Qiu SJ, Dai Z, He $\mathrm{R}$ et al. PKM2 promotes metastasis by recruiting myeloidderived suppressor cells and indicates poor prognosis for hepatocellular carcinoma. Oncotarget. 2015; 6:846-61.

19. Castillo SD, Angulo B, Suarez-Gauthier A, Melchor L, Medina PP, Sanchez-Verde L, Torres-Lanzas J, Pita G, Benitez J and Sanchez-Cespedes M. Gene amplification of the transcription factor DP1 and CTNND1 in human lung cancer. J Pathol. 2010; 222:89-98.

20. Wang Y, Wen M, Kwon Y, Xu Y, Liu Y, Zhang P, He X, Wang Q, Huang Y, Jen KY, LaBarge MA, You L, Kogan SC et al. CUL4A induces epithelial-mesenchymal transition and promotes cancer metastasis by regulating ZEB1 expression. Cancer Res. 2014; 74:520-31.

21. Fu J, Lv X, Lin H, Wu L, Wang R, Zhou Z, Zhang B, Wang YL, Tsang BK, Zhu C and Wang H. Ubiquitin ligase cullin 7 induces epithelial-mesenchymal transition in human choriocarcinoma cells. J Biol Chem. 2010; 285:10870-9.

22. Ang YS, Tsai SY, Lee DF, Monk J, Su J, Ratnakumar K, Ding J, Ge Y, Darr H, Chang B, Wang J, Rendl M, Bernstein E et al. Wdr5 mediates self-renewal and reprogramming via the embryonic stem cell core transcriptional network. Cell. 2011; 145:183-97.

23. Ohta K, Haraguchi N, Kano Y, Kagawa Y, Konno M, Nishikawa S, Hamabe A, Hasegawa S, Ogawa H, Fukusumi T, Uemura M, Nishimura J, Hata T et al. Depletion of JARID1B induces cellular senescence in human colorectal cancer. Int J Oncol. 2013; 42:1212-8.

24. Wang H, Quah SY, Dong JM, Manser E, Tang JP and Zeng Q. PRL-3 down-regulates PTEN expression and signals through PI3K to promote epithelial-mesenchymal transition. Cancer Res. 2007; 67:2922-6.

25. Kim SR, Lee KS, Park SJ, Min KH, Lee KY, Choe YH, Lee YR, Kim JS, Hong SJ and Lee YC. PTEN down-regulates IL-17 expression in a murine model of toluene diisocyanateinduced airway disease. J Immunol. 2007; 179:6820-9.

26. Secombe J and Eisenman RN. The function and regulation of the JARID1 family of histone $\mathrm{H} 3$ lysine 4 demethylases: the Myc connection. Cell Cycle. 2007; 6:1324-8.

27. Albert M, Schmitz SU, Kooistra SM, Malatesta M, Morales Torres C, Rekling JC, Johansen JV, Abarrategui I and Helin $\mathrm{K}$. The histone demethylase Jarid1b ensures faithful mouse development by protecting developmental genes from aberrant H3K4me3. PLoS Genet. 2013; 9:e1003461.

28. Duns G, van den Berg E, van Duivenbode I, Osinga J, Hollema H, Hofstra RM and Kok K. Histone methyltransferase gene SETD2 is a novel tumor suppressor gene in clear cell renal cell carcinoma. Cancer Res. 2010; 70:4287-91.
29. Hung SY, Lin HH, Yeh KT and Chang JG. Histonemodifying genes as biomarkers in hepatocellular carcinoma. Int J Clin Exp Pathol. 2014; 7:2496-507.

30. Wang X, Ju L, Fan J, Zhu Y, Liu X, Zhu K, Wu M and Li L. Histone H3K4 methyltransferase M1l1 regulates protein glycosylation and tunicamycin-induced apoptosis through transcriptional regulation. Biochim Biophys Acta. 2014; 1843:2592-602.

31. Rodriguez-Paredes M, Martinez de Paz A, Simo-Riudalbas L, Sayols S, Moutinho C, Moran S, Villanueva A, VazquezCedeira M, Lazo PA, Carneiro F, Moura CS, Vieira J, Teixeira MR et al. Gene amplification of the histone methyltransferase SETDB1 contributes to human lung tumorigenesis. Oncogene. 2014; 33:2807-13.

32. Kuzbicki L, Lange D, Straczynska-Niemiec A and Chwirot BW. JARID1B expression in human melanoma and benign melanocytic skin lesions. Melanoma Res. 2013; 23:8-12.

33. Radberger P, Radberger A, Bykov VJ, Seregard S and Economou MA. JARID1B protein expression and prognostic implications in uveal melanoma. Invest Ophthalmol Vis Sci. 2012; 53:4442-9.

34. Lu W, Liu S, Li B, Xie Y, Adhiambo C, Yang Q, Ballard BR, Nakayama KI, Matusik RJ and Chen Z. SKP2 inactivation suppresses prostate tumorigenesis by mediating JARID1B ubiquitination. Oncotarget. 2015; 6:771-88.

35. Xie Y, Liu S, Lu W, Yang Q, Williams KD, Binhazim AA, Carver BS, Matusik RJ and Chen Z. Slug regulates E-cadherin repression via p19Arf in prostate tumorigenesis. Mol Oncol. 2014; 8:1355-64.

36. Mulholland DJ, Kobayashi N, Ruscetti M, Zhi A, Tran LM, Huang J, Gleave $\mathrm{M}$ and $\mathrm{Wu} \mathrm{H}$. Pten loss and RAS/MAPK activation cooperate to promote EMT and metastasis initiated from prostate cancer stem/progenitor cells. Cancer Res. 2012; 72:1878-89.

37. Davis NM, Sokolosky M, Stadelman K, Abrams SL, Libra M, Candido S, Nicoletti F, Polesel J, Maestro R, D’Assoro A, Drobot L, Rakus D, Gizak A et al. Deregulation of the EGFR/PI3K/PTEN/Akt/mTORC1 pathway in breast cancer: possibilities for therapeutic intervention. Oncotarget. 2014; 5:4603-50.

38. Ohta K, Hoshino H, Wang J, Ono S, Iida Y, Hata K, Huang SK, Colquhoun S and Hoon DS. MicroRNA-93 activates c-Met/PI3K/Akt pathway activity in hepatocellular carcinoma by directly inhibiting PTEN and CDKN1A. Oncotarget. 2014. 